\title{
Factors determining outcomes in adult patients operated for congenital heart diseases
}

\author{
Sachin Talwar", Manikala Vinod Kumar, Shiv Kumar Choudhary, Balram Airan \\ From World Society of Cardiothoracic Surgeons 25th Anniversary Congress, Edinburgh \\ Edinburgh, UK. 19-22 September 2015
}

\section{Background/Introduction}

Older patients with congenital heart disease $(\mathrm{GUCH})$ present a unique challenge.

\section{Aims/Objectives}

To analyze factors predicting early cardiac morbidity in $\mathrm{GUCH}$ at a tertiary care centre.

\section{Method}

Between January 2004-December 2014, 1432 patients $\leq 13$ years of age underwent surgery for GUCH. Factors associated with early cardiac morbidity were analyzed.

\section{Results}

On multivariate analysis, previous sternotomy, aortic cross clamp time more than $45 \mathrm{~min}$, cyanosis, emergency procedure were identified as independent predictors of early cardiac morbidity with respective odds ratios of 10.5, 3.7, 2.3 and 8.0. These four variables together could discriminate $77 \%$ of all procedures correctly as to their immediate post-operative morbidity. Taking the log odds with each of these 4 as the respective weights, a score was generated. The weights were previous sternotomy (2.4), aortic cross clamp $>45 \mathrm{~min}$ (1.3), emergency (2.1), cyanosis (0.8), if the respective condition is present, zero otherwise. The score ranged from 0 to 4.5.The average value of the score based on the 4 variables was significantly higher in cases with cardiac morbidity $(0.75 \pm 0.88) \mathrm{v} / \mathrm{s}(1.85 \pm 1.17), \mathrm{p}<0.001$. Distribution of the scores was significantly different between patients with and without morbidity. $67 \%$ patients without any morbidity had score $<1$ compared to $24.6 \%$ with morbidity. Only $1.2 \%$ patients without morbidity had score of $\leq 3$ compared to $15 \%$ patients

Department of Cardiothoracic and Vascular Surgery, Cardiothoracic Sciences Centre, India Institute of Medical Sciences, Ansari, Nagar, New Delhi, 110029, India 